\title{
Weed Control, Environmental Impact and Profitability of Weed Management Strategies in Glyphosate-Resistant Corn
}

\author{
Nader Soltani $^{1 *}$, Christie L. Stewart ${ }^{2}$, Robert E. Nurse ${ }^{3}$, Laura L. Van Eerd ${ }^{1}$, Richard J. Vyn ${ }^{1}$, \\ Peter H. Sikkema ${ }^{1}$ \\ ${ }^{1}$ Ridgetown Campus, University of Guelph, Ridgetown, Canada $;{ }^{2}$ Western University, London, Canada; ${ }^{3}$ Agriculture and Agri-Food \\ Canada, Harrow, Canada. \\ Email: ${ }^{*}$ soltanin@uoguelph.ca
}

Received August $15^{\text {th }}, 2012$; revised September $14^{\text {th }}, 2012$; accepted October $11^{\text {th }}, 2012$

\begin{abstract}
Eleven field trials were conducted over a three-year period (2006-2008) at three locations in southwestern Ontario, Canada to evaluate the effect of various weed management strategies in glyphosate-resistant corn on weed control, crop injury, corn yield, environmental impact and profit margin. No visible injury resulted from the herbicide treatments evaluated. Overall, the effect of all factors assessed were location specific. By 56 days after treatment, depending on location, glyphosate applied at the 7-8 leaf stage (LPOST), preemergence (PRE) herbicides followed by $(f b)$ glyphosate LPOST and sequential glyphosate applications (EPOST (3-4 leaf stage) followed by LPOST) provided more consistent control of annual broadleaf weeds and annual grasses compared to glyphosate applied alone EPOST. Weed control at 56 days after treatment was lower when glyphosate was applied alone LPOST compared to sequential applications of glyphosate or PRE herbicides $f b$ glyphosate. There were no differences in corn yield among the sequential programs evaluated; however, a yield benefit was found when a sequential program was used compared to glyphosate applied alone LPOST. Among the sequential programs the lowest environmental impact was isoxaflutole/atrazine $f b$ glyphosate. The lowest profit margins were associated with atrazine, S-metolachlor/atrazine/benoxacor, dicamba/atrazine and glyphosate LPOST treatments compared to all other treatments. Overall, profit margins tended to be somewhat higher for treatments that included glyphosate applications. Based on these results, the most efficacious and profitable weed management program in corn was a sequential application of glyphosate; however, isoxaflutole/atrazine $f b$ glyphosate was the treatment with the lowest environmental risk while also adding glyphosate stewardship benefits.
\end{abstract}

Keywords: Environmental Impact Quotient (EIQ); Glyphosate; Profit Margin; Corn; Zea mays L.

\section{Introduction}

The demand on growers to economically produce environmentally sustainable food while maintaining herbicide stewardship is increasing. To achieve this goal, data on weed control, crop yield, economics and environmental impacts of herbicides are needed to help identify the most advantageous herbicide program. Growers of glyphosate-resistant corn have several weed management options, including pre-emergence (PRE), post-emergence (POST), tankmixes and sequential applications. Traditional management strategies for corn have included atrazine $[1,2]$; however the future of atrazine use in corn is unclear $[3,4]$. Since the introduction of glyphosate resistant corn, one- or two-pass glyphosate-only applications are now options that can simplify weed manage-

${ }^{*}$ Corresponding author. ment and can be an effective method used to improve weed control [2,5-7]. However, sole reliance on glyphosate increases weed selection pressure, potentially selecting for glyphosate-resistant weeds [2,8-10]. Tank-mixes or sequential applications that utilize more than one herbicide modes of action can reduce selection pressure [2].

Timing of herbicide application is also critical for effective weed control [11-14]. A single-pass herbicide program, PRE or POST can result in weed escapes if the program fails to control all weeds or has no soil residual $[13,15]$. Weed escapes can be more difficult to control due to increased size, resulting in reduced herbicide efficacy of rescue sprays $[16,17]$. Later germinating weeds are of particular concern with a single-pass application of glyphosate because of its lack of residual control $[6,18]$. Sequential in-crop applications of glyphosate or combining a residual PRE herbicide with a POST application 
can improve control of late-emerging weeds [5,6,18-22].

To increase sustainability, management decisions need to include an assessment of the environmental impact of weed control strategies. By using lower herbicide application rates and/or safer products, the environmental impact of weed control may be reduced. Based on toxicological and physicochemical properties of pesticides, the environmental impact quotient (EIQ) measures the relative potential risk of pesticide active ingredients on human and ecological health based on three risk components: farm worker, consumer, and environment [23,24]. A higher environmental impact (EI) indicates a greater risk of detrimental impact. The EIQ was designed to provide growers and other decision makers with one number that indicates the magnitude of relative risk of different pesticides [25-27].

Current economic pressures facing growers require that weed management programs have high efficacy while providing positive economic returns. The cost of herbicides used in a weed management program, along with their application costs, needs to be offset by profits gained from crop yields otherwise the management program is not advantageous. Economic loss can result from a failed weed management program, leading to increased weed competition and yield losses [28,29]. Weed control failure may require a rescue spray, thereby increasing costs and decreasing a grower's profit margin. Herbicide-resistant crop use is argued to provide greater economic benefits to growers compared to non-transgenic crops due to greater weed control and reduced inputs costs [30]. Yet studies by Bradley et al. [2], Ferrell and Witt [31] and Johnson et al. [29] do not indicate an increase in profitability with the use of glyphosate-resistant corn.
To justify the most appropriate weed management strategy, the decision-making process must include an assessment of herbicide efficacy, environmental impact and economic profitability, while recognizing that tradeoffs among those factors will occur. Therefore, the objective of this study is to determine which herbicide strategy for glyphosate-resistant corn will be most efficacious and economically profitable while providing low environmental impact.

\section{Material and Methods}

\subsection{Site Descriptions and Procedures}

Eleven field trials were conducted in southwestern Ontario at the Greenhouse and Processing Crops Research Centre, Agriculture and Agri-Food Canada, Harrow, Ontario in 2007 and 2008, at the Huron Research Station, Exeter, Ontario in 2006, 2007 and 2008 and two different sites at the University of Guelph, Ridgetown Campus, Ridgetown, Ontario in 2006, 2007 and 2008 (RCA, Site 1 and RCB, Site 2). Soil descriptions from each location can be found in Table 1.

Procedures at all sites were the same unless otherwise noted. Experiments were arranged in a randomized complete block design with four replicates. There were a total of thirteen treatments: a non-treated weedy control, a weed-free control, atrazine $\left(1000 \mathrm{~g} \cdot \mathrm{ai} \cdot \mathrm{ha}^{-1}\right)$, s-metolachlor/atrazine/benoxacor $\left(1080 \mathrm{~g} \cdot \mathrm{ai} \cdot \mathrm{ha}^{-1}\right)$, isoxaflutole $\left(40 \mathrm{~g} \cdot \mathrm{ai} \cdot \mathrm{ha}^{-1}\right)+$ atrazine $\left(400 \mathrm{~g} \cdot \mathrm{ai} \cdot \mathrm{ha}^{-1}\right)$, dicamba/atrazine (1000 g.ai $\left.\cdot \mathrm{ha}^{-1}\right)$, glyphosate $\left(900 \mathrm{~g} \cdot \mathrm{ae} \cdot \mathrm{ha}^{-1} ; 3\right.$ - 4 leaf stage, EPOST), glyphosate (900 g.ae $\cdot \mathrm{ha}^{-1} ; 7$ - 8 leaf stage, LPOST), dicamba/atrazine $\left(1000 \mathrm{~g} \cdot \mathrm{ai} \cdot \mathrm{ha}^{-1}\right)$ followed by $(f b)$ glyphosate $\left(900 \mathrm{~g} \cdot \mathrm{ae} \cdot \mathrm{ha}^{-1}\right)$, atrazine $(1000$ $\left.\mathrm{g} \cdot \mathrm{ai} \cdot \mathrm{ha}^{-1}\right) \mathrm{fb}$ LPOST glyphosate $\left(900 \mathrm{~g} \cdot \mathrm{ae} \cdot \mathrm{ha}^{-1}\right)$, s-meto-

Table 1. Soil characteristics at Harrow (2007, 2008), Exeter (2006, 2007, 2008) and Ridgetown (2006, 2007, 2008$)$, Ontario.

\begin{tabular}{|c|c|c|c|c|c|c|c|}
\hline Location & Year & Soil pH & Organic matter \% & Soil texture & Sand & Silt \% & Clay \\
\hline \multirow{2}{*}{ Harrow } & 2007 & 6.0 & 2.6 & Fox Sandy Loam & 82.5 & 5.0 & 12.5 \\
\hline & 2008 & 6.0 & 2.6 & Fox Sandy Loam & 82.5 & 5.0 & 12.5 \\
\hline \multirow{3}{*}{ Exeter } & 2006 & 7.9 & 3.4 & Brookston Clay Loam & 33.0 & 35.0 & 32.0 \\
\hline & 2007 & 7.8 & 3.7 & Brookston Clay Loam & 38.0 & 41.0 & 21.0 \\
\hline & 2008 & 7.9 & 3.0 & Brookston Clay Loam & 34.0 & 33.0 & 33.0 \\
\hline \multirow{3}{*}{$\mathrm{RCA}$} & 2006 & 6.7 & 5.9 & Clay Loam & 35.3 & 34.9 & 29.9 \\
\hline & 2007 & 7.0 & 4.0 & Sandy Clay Loam & 55.3 & 24.2 & 20.5 \\
\hline & 2008 & 7.4 & 5.0 & Loam & 42.9 & 32.8 & 24.4 \\
\hline \multirow{3}{*}{$\mathrm{RCB}$} & 2006 & 7.4 & 5.0 & Loam & 42.9 & 32.8 & 24.4 \\
\hline & 2007 & 6.9 & 4.8 & Very Fine Sandy Loam & 63.1 & 19.3 & 17.6 \\
\hline & 2008 & 6.8 & 5.3 & Sandy Clay Loam & 52.4 & 26.3 & 21.3 \\
\hline
\end{tabular}

Abbreviations: RCA, Ridgetown Site 1; RCB, Ridgetown Site 2. 
lachlor/atrazine/benoxacor (1080 $\left.\mathrm{g} \cdot \mathrm{ai} \cdot \mathrm{ha}^{-1}\right) \mathrm{fb}$ LPOST glyphosate $\left(900 \mathrm{~g} \cdot \mathrm{ae}^{\mathrm{h}} \cdot \mathrm{ha}^{-1}\right)$, isoxaflutole $\left(40 \mathrm{~g} \cdot \mathrm{ai}^{-1} \cdot \mathrm{ha}^{-1}\right)+$ atrazine $\left(400 \mathrm{~g} \cdot \mathrm{ai} \cdot \mathrm{ha}^{-1}\right)$ fb LPOST glyphosate (900 $\left.\mathrm{g} \cdot \mathrm{ae} \cdot \mathrm{ha}^{-1}\right)$, EPOST glyphosate $\left(900 \mathrm{~g} \cdot \mathrm{ae} \cdot \mathrm{ha}^{-1}\right) \mathrm{fb}$ LPOST glyphosate $\left(900 \mathrm{~g} \cdot \mathrm{ae} \cdot \mathrm{ha}^{-1}\right)$.

Each treatment plot was $3 \mathrm{~m}$ ( 4 corn rows) wide by 8 $\mathrm{m}$ long at Harrow, $3 \mathrm{~m}$ by $10 \mathrm{~m}$ at Exeter and $2 \mathrm{~m}$ by $8 \mathrm{~m}$ at both sites in Ridgetown. Glyphosate-resistant corn hybrids [Pioneer 36W68; 2008, Pioneer 36W69 (Harrow); 2006, Pioneer 38H65; 2007, Pioneer 38B86; 2008, Pioneer 38M68 (Exeter); 2006, Pioneer 38H69; 2007, Pioneer 38W69; 2008, Pioneer 35F44 (Ridgetown)] were seeded at a density of at least 71,000 seeds $\cdot \mathrm{ha}^{-1}$ in rows spaced $75 \mathrm{~cm}$ apart. Herbicides were applied using a $\mathrm{CO}_{2}$ - pressurized sprayer calibrated to deliver $222 \mathrm{~L} \cdot \mathrm{ha}^{-1}$ aqueous solution at $210 \mathrm{kPa}$ using flat fan 110-03 XR nozzles $\left(\right.$ TeeJet $^{\circledR}$ flat fan 11003 XR nozzles, Spraying Systems Company, P.O. Box 7900 Wheaton, IL 601897900 ) in 2007 and ULD 120-02 nozzles (VeeJet ${ }^{\circledR}$ Ultra low-drift 12002 nozzles, Spraying Systems Company, P.O. Box 7900 Wheaton, IL 60189-7900) in 2008 spaced $50 \mathrm{~cm}$ apart at Harrow, $200 \mathrm{~L} \cdot \mathrm{ha}^{-1}$ aqueous solution at $241 \mathrm{kPa}$ using flat fan 8002 VS nozzles (TeeJet ${ }^{\circledR}$ flat fan 8002 VS nozzles, Spraying Systems Company, P.O. Box 7900 Wheaton, IL 60189-7900) in 2006 and 2007 and ULD 120-02 nozzles (2008) spaced $50 \mathrm{~cm}$ apart at Exeter and $200 \mathrm{~L} \cdot \mathrm{ha}^{-1}$ aqueous solution at $207 \mathrm{kPa}$ using ULD 120-02 nozzles spaced $50 \mathrm{~cm}$ apart at Ridgetown.

Crop injury was estimated visually 7, 14 and 28 days after treatment (DAT), using a scale of 0 to $100 \%$ where a rating of 0 was defined as no visible plant injury and a rating of 100 was defined as plant death. Percent weed control was visually assessed 28 and 56 DAT using a scale of $0 \%$ to $100 \%$ where a rating of 0 was defined as no visible weed control and a rating of 100 was defined as complete control. Only data from 56 DAT are presented in this manuscript. Corn was mechanically harvested at physiological maturity using a plot combine at all sites. Corn yields were adjusted to a $15.5 \%$ moisture level.

\subsection{Statistical Analyses}

All data were subjected to analysis of variance and analyzed using the PROC MIXED procedure in SAS statistical software (Version 8. SAS Institute, Inc., Box 8000, SAS Circle, Cary, NC 27512). Variances were partitioned into the fixed effect of herbicide treatment and into the random effects of environment (year and location). When there was no significant interaction between environment and treatment the data were pooled. Contrast comparisons among herbicide application timings represent $a$ priori orthoganol contrasts. The assumptions of the variance analysis were tested by ensuring that the residuals were random, homogeneous, with a normal distribution about a mean of zero using residual plots and a Shapiro-Wilk normality test. All percentage data required an arcsine square root transformation. Yield data did not require transformation. All percentage data presented in tables are on the back-transformed scale. Treatment means were separated at the $5 \%$ level of significance using a Fisher's Protected LSD test.

\subsection{Environmental Impact}

The environmental risk for each herbicide treatment was determined using published EIQ values for all active ingredients (a.i.) [24]. However, the EIQ for atrazine, metolachlor, and isoxaflutole were recalculated based on PRE vs POST application, where the plant surface persistence value $(\mathrm{P})$ of 1 was used instead of 3 , respectively. The environmental impact of each treatment was calculated by multiplying herbicide EIQ by the amount applied in $\mathrm{kg} \cdot \mathrm{ai} / \mathrm{ae} \cdot \mathrm{ha}^{-1}$. For herbicide products and/or tank mixes that contain more than one a.i., the EI was calculated by summing EIQs at the appropriate proportion.

\subsection{Profitability Analysis}

The profitability analysis is based on the level of profit margins over weed control costs, measured as gross income less herbicide and application costs. Gross income for each replication was calculated as the yield multiplied by average price for corn between 2006 and 2008, based on the claim prices reported by Agricorp (Agricorp, 1 Stone Road West, Box 3660 Station Central, Guelph, ON $\mathrm{N} 1 \mathrm{H}$ 8M4, Canada). Herbicide costs for each treatment are based on the 2007 herbicide prices reported by AGRIS (AGRIS Co-operative Ltd., 835 Park Avenue West, Chatham, ON N7M 5J6, Canada). Application costs are determined based on cost of production data reported by the Ontario Ministry of Agriculture, Food and Rural Affairs (Field Crop Budgets, Publication 60, updated annually; Ontario Ministry of Agriculture, Food and Rural Affairs, 1 Stone Road West, Guelph, ON N1G 4Y2, Canada). All other costs of production are assumed to be constant across treatments, thus they are not considered in the analysis. Pairwise comparisons are made between treatments using SPSS (SPSS Software, Version 16.0 SPSS Inc., 233 S. Wacher Drive, Chicago, IL 60606) to test for significant differences in average profit margins between treatments. These pairwise comparisons are made across all locations and years as well as for each location (RCA, RCB, Exeter, and Harrow) in each year. This allows for testing for overall differences in profitability between treatments as well as for testing for variations in relative profitability for specific treatments between different locations, between different years, and 
between different location-years. If such variations are found to exist, this would suggest that profit-maximizing weed control methods may vary under different circumstances.

\section{Results and Discussion}

\subsection{Weed Control}

The dominant weed species in this study were redroot pigweed, common ragweed, common lambsquarters and velvetleaf. Because annual grass species varied by location, all species were grouped together for analysis.

\subsubsection{Redroot Pigweed}

All treatments provided greater than $90 \%$ control of redroot pigweed (Table 2). When glyphosate was applied
EPOST redroot pigweed control was reduced by $7 \%$ compared to LPOST glyphosate, a PRE herbicide $f b$ glyphosate or a sequential glyphosate application. Stewart et al. [7] also previously demonstrated that an EPOST application of glyphosate provided 7\% - 11\% lower redroot pigweed control compared to a sequential application of glyphosate in corn. In contrast, a sequential application of glyphosate did not improve redroot pigweed control in comparison to a single EPOST application in soybean [13]. Nurse et al. [6] reported a decrease of 35.9 plants $\mathrm{m}^{-2}$ of redroot pigweed when flufenacet + metribuzin $f b$ glyphosate was applied compared to when glyphosate was applied alone in corn. The results of several other studies support that late-emerging weeds are controlled most effectively using sequential applications of glyphosate or by following a PRE herbicide with a

Table 2. Mean percent control of AMARE in response to weed management strategies 56 days after treatment at Exeter and Ridgetown, ON from 2006 to 2008 and Harrow, ON from 2007 to $2008^{a}$.

\begin{tabular}{|c|c|c|c|}
\hline \multirow{2}{*}{ Treatment } & \multirow{2}{*}{ Timing } & \multirow{2}{*}{$\begin{array}{c}\text { Rate } \\
\mathbf{g} \cdot \mathbf{a i} / \mathbf{a e} \cdot \mathbf{h a}{ }^{-1}\end{array}$} & \multirow{2}{*}{$\begin{array}{c}\text { Weed control (\%) } \\
\text { Pooled }\end{array}$} \\
\hline & & & \\
\hline Weed-free check & & & $100 \mathrm{a}$ \\
\hline Atrazine & PRE & 1000 & $98 \mathrm{a}$ \\
\hline s-metolachlor/atrazine/benoxacor & PRE & 1080 & $90 \mathrm{~b}$ \\
\hline Isoxaflutole + atrazine & $\mathrm{PRE}+\mathrm{PRE}$ & $40+400$ & $99 \mathrm{a}$ \\
\hline Dicamba/atrazine & PRE & 1000 & $100 \mathrm{a}$ \\
\hline Glyphosate & EPOST & 900 & $93 b$ \\
\hline Glyphosate & LPOST & 900 & $100 \mathrm{a}$ \\
\hline Dicamba/atrazine $f b$ glyphosate & PRE $f b$ LPOST & $1000 f b 900$ & $100 \mathrm{a}$ \\
\hline Atrazine $f b$ glyphosate & PRE $f b$ LPOST & $1000 f b 900$ & $100 \mathrm{a}$ \\
\hline s-metolachlor/atrazine/benoxacor $f b$ glyphosate & PRE $f b$ LPOST & $1080 f b 900$ & $100 \mathrm{a}$ \\
\hline Isoxaflutole + atrazine $f b$ glyphosate & PRE+PRE $f b$ LPOST & $40+400 f b 900$ & $100 \mathrm{a}$ \\
\hline Glyphosate $f b$ glyphosate & EPOST $f b$ LPOST & $900 f b 900$ & $100 \mathrm{a}$ \\
\hline \multicolumn{4}{|l|}{ Contrasts $^{\mathrm{b}}$} \\
\hline WF vs glyphosate EPOST & & & $*$ \\
\hline WF vs glyphosate LPOST & & & NS \\
\hline Glyphosate EPOST vs PRE $f b$ glyphosate LPOST & & & $*$ \\
\hline Glyphosate LPOST vs PRE $f b$ glyphosate LPOST & & & NS \\
\hline Glyphosate EPOST $f b$ glyphosate LPOST vs PRE $f b$ glyphosate LPOST & & & NS \\
\hline Glyphosate EPOST vs glyphosate EPOST $f b$ glyphosate LPOST & & & $*$ \\
\hline Glyphosate LPOST vs glyphosate EPOST $f b$ glyphosate LPOST & & & NS \\
\hline Glyphosate EPOST vs glyphosate LPOST & & & * \\
\hline
\end{tabular}

${ }^{a}$ Data were pooled by environment (location and year) when the interaction between environment and treatment was non-significant. Means are presented on the back-transformed scale. Means followed by the same letter within a column are not significantly different according to Fisher's Protected LSD (P $<0.05$ ); ${ }^{\mathrm{b}}$ a priori orthogonal contrasts; $*=$ significant $(\mathrm{P}<0.05)$. Abbreviations: AMARE, redroot pigweed; PRE, preemergence; POST, postemergence; EPOST, early postemergence; LPOST, late postemergence; RCA, Ridgetown Site 1; RCB, Ridgetown Site 2; WF, weed-free; fb, followed by; NS, not significant 
POST application of glyphosate $[6,18,20]$.

\subsubsection{Common Ragweed}

Preemergence application of S-metolachlor/atrazine/benoxacor provided less than $77 \%$ control of common ragweed except at RCA in 2007 (Table 3). Furthermore, when applied alone, atrazine PRE had no control of common ragweed at RCA in 2006 and less than 30\% control at RCB in 2006 and 2007. By 56 DAT common ragweed may have escaped the soil residual provided by atrazine, resulting in the poor control observed with $S$ metolachlor/benoxacor/atrazine or atrazine alone at these locations. Glyphosate applied LPOST had as much as $17 \%$ higher common ragweed control at Exeter in 2006 compared to when glyphosate was applied EPOST. This is most likely due to common ragweed emerging after the EPOST application. Sequential applications of glyphosate increased common ragweed control compared to glyphosate applied EPOST by $19 \%, 2 \%, 22 \%$, and $2 \%$ at RCA, RCB, and Exeter in 2006 and RCB in 2007, respectively (Table 3). Sequential glyphosate application also increased common ragweed control by $21 \%$ and $5 \%$ at RCA 2006 and Exeter 2007, respectively, compared to a LPOST application of glyphosate. Generally, the appli-

Table 3. Mean percent control of AMBEL in response to several weed management strategies 56 days after treatment at Exeter and Ridgetown, ON from 2006 to 2008 and Harrow, ON, 2008

\begin{tabular}{|c|c|c|c|c|c|c|c|c|c|}
\hline \multirow{3}{*}{ Treatment } & \multirow{3}{*}{ Timing } & \multirow{3}{*}{$\frac{\text { Rate }}{\mathrm{g} \cdot \mathbf{a i} / \mathbf{a e} \cdot \mathbf{h a}{ }^{-1}}$} & \multicolumn{7}{|c|}{ Weed Control \% } \\
\hline & & & \multicolumn{3}{|c|}{2006} & \multicolumn{3}{|c|}{2007} & \multirow{2}{*}{$\begin{array}{c}2008 \\
\text { Pooled }\end{array}$} \\
\hline & & & RCA & RCB & Exeter & RCA & RCB & Exeter & \\
\hline \multicolumn{3}{|l|}{ Weed-free check } & $100 \mathrm{a}$ & $100 \mathrm{a}$ & $100 \mathrm{a}$ & $100 \mathrm{a}$ & $100 \mathrm{a}$ & $100 \mathrm{a}$ & $100 \mathrm{a}$ \\
\hline Atrazine & PRE & 1000 & $0 \mathrm{e}$ & $26 \mathrm{~b}$ & $100 \mathrm{a}$ & $96 \mathrm{e}$ & $29 \mathrm{e}$ & $99 \mathrm{ab}$ & $65 b$ \\
\hline s-metolachlor/atrazine/benoxacor & PRE & 1080 & $0 \mathrm{e}$ & $44 b$ & $74 \mathrm{c}$ & $97 d$ & $25 \mathrm{e}$ & $77 \mathrm{c}$ & $51 \mathrm{~b}$ \\
\hline Isoxaflutole + atrazine & $\mathrm{PRE}+\mathrm{PRE}$ & $40+400$ & $78 \mathrm{~d}$ & $97 \mathrm{a}$ & $100 \mathrm{a}$ & $99 \mathrm{~b}$ & $63 \mathrm{~d}$ & $100 \mathrm{a}$ & $99 \mathrm{a}$ \\
\hline Dicamba/atrazine & PRE & 1000 & $99 \mathrm{ab}$ & $99 \mathrm{a}$ & $100 \mathrm{a}$ & $99 \mathrm{~b}$ & $99 \mathrm{ab}$ & $100 \mathrm{a}$ & $100 \mathrm{a}$ \\
\hline Glyphosate & EPOST & 900 & $81 \mathrm{~d}$ & $98 \mathrm{a}$ & $78 \mathrm{c}$ & $99 \mathrm{~b}$ & $97 \mathrm{c}$ & $100 \mathrm{a}$ & $98 \mathrm{a}$ \\
\hline Glyphosate & LPOST & 900 & $79 \mathrm{~d}$ & $97 \mathrm{a}$ & $95 \mathrm{~b}$ & $98 \mathrm{c}$ & $98 \mathrm{bc}$ & $95 \mathrm{~b}$ & $99 \mathrm{a}$ \\
\hline Dicamba/atrazine $f b$ glyphosate & PRE $f b$ LPOST & $1000 f b 900$ & $97 \mathrm{bc}$ & $99 \mathrm{a}$ & $100 \mathrm{a}$ & $99 \mathrm{~b}$ & $99 \mathrm{ab}$ & $100 \mathrm{a}$ & $100 \mathrm{a}$ \\
\hline Atrazine $f b$ glyphosate & PRE $f b$ LPOST & $1000 \mathrm{fb} 900$ & $98 \mathrm{bc}$ & $98 \mathrm{a}$ & $100 \mathrm{a}$ & $99 \mathrm{~b}$ & $99 \mathrm{ab}$ & $100 \mathrm{a}$ & $100 \mathrm{a}$ \\
\hline $\begin{array}{l}\text { s-metolachlor/atrazine/benoxacor } \\
\text { fb glyphosate }\end{array}$ & PRE $f b$ LPOST & $1080 f b 900$ & $94 \mathrm{c}$ & $98 \mathrm{a}$ & $99 \mathrm{ab}$ & $98 \mathrm{c}$ & $99 \mathrm{ab}$ & $97 \mathrm{ab}$ & $99 \mathrm{a}$ \\
\hline Isoxaflutole + atrazine $f b$ glyphosate & PRE + PRE $f b$ LPOST & $40+400 f b 900$ & $100 \mathrm{a}$ & $99 \mathrm{a}$ & $100 \mathrm{a}$ & $99 \mathrm{~b}$ & $99 \mathrm{ab}$ & $100 \mathrm{a}$ & $100 \mathrm{a}$ \\
\hline Glyphosate $f b$ glyphosate & EPOST $f b$ LPOST & $900 f b 900$ & $100 \mathrm{a}$ & $99 \mathrm{a}$ & $99 \mathrm{ab}$ & $99 \mathrm{~b}$ & $99 \mathrm{ab}$ & $100 \mathrm{a}$ & $100 \mathrm{a}$ \\
\hline \multicolumn{10}{|l|}{ Contrasts $^{\mathrm{b}}$} \\
\hline \multicolumn{3}{|l|}{ WF vs glyphosate EPOST } & $*$ & * & $*$ & * & * & NS & NS \\
\hline \multicolumn{3}{|l|}{ WF vs glyphosate LPOST } & $*$ & * & * & * & * & * & NS \\
\hline \multicolumn{3}{|c|}{ Glyphosate EPOST vs PRE $f b$ glyphosate LPOST } & * & * & * & * & * & NS & NS \\
\hline \multicolumn{3}{|c|}{ Glyphosate LPOST vs PRE $f b$ glyphosate LPOST } & $*$ & NS & * & * & NS & * & NS \\
\hline \multicolumn{3}{|c|}{ Glyphosate EPOST $f b$ glyphosate LPOST vs PRE $f b$ glyphosate LPOST } & NS & NS & NS & NS & NS & NS & NS \\
\hline \multicolumn{3}{|c|}{ Glyphosate EPOST vs glyphosate EPOST $f b$ glyphosate LPOST } & $*$ & $*$ & * & NS & * & NS & NS \\
\hline \multicolumn{3}{|c|}{ Glyphosate LPOST vs glyphosate EPOST $f b$ glyphosate LPOST } & * & NS & NS & NS & NS & * & NS \\
\hline \multicolumn{3}{|c|}{ Glyphosate EPOST vs glyphosate LPOST } & NS & * & * & NS & * & * & NS \\
\hline
\end{tabular}

${ }^{\mathrm{a}}$ Data were pooled by environment (location and year) when the interaction between environment and treatment was non-significant. Means are presented on the back-transformed scale. Means followed by the same letter within a column are not significantly different according to Fisher's Protected LSD (P $<0.05$ ); ${ }^{\mathrm{b}}$ a priori orthogonal contrasts. $*=$ significant $(\mathrm{P}<0.05)$. Abbreviations: AMBEL, common ragweed, PRE, preemergence; POST, postemergence; EPOST, early postemergence; LPOST, late postemergence; RCA, Ridgetown Site 1; RCB, Ridgetown Site 2; WF, weed-free; $f b$, followed by; NS, not significant. 
cation of a PRE herbicide $f b$ glyphosate improved common ragweed control compared to a one-pass application of glyphosate applied EPOST or LPOST, but not a sequential application of glyphosate. This is supported by Nurse et al. [6] who found that common ragweed control was improved with a sequential application of flufenacet + metribuzin $f b$ glyphosate in comparison to a single application of glyphosate in corn.

\subsubsection{Common Lambsquarters}

Glyphosate applied EPOST had lower common lambsquarters control compared to a PRE herbicide $f b$ glyphosate in 2006 at Exeter and RCA, in 2007 at all locations and in 2008 within Environment 1 (Table 4). Similarly, glyphosate applied LPOST had lower common lambsquarters control compared to a PRE herbicide $f b$ glyphosate in 2006 at Exeter and RCA, in 2007 at Exeter

Table 4. Mean percent control of CHEAL in response to several weed management strategies 56 days after treatment at Exeter and Ridgetown, ON from 2006 to 2008 and Harrow, ON from 2007 to $2008^{\mathrm{a}}$.

\begin{tabular}{|c|c|c|c|c|c|c|c|c|c|c|c|}
\hline \multirow{3}{*}{ Treatment } & \multirow{3}{*}{ Timing } & \multirow[t]{3}{*}{ Rate } & \multicolumn{9}{|c|}{ Weed Control \% } \\
\hline & & & \multicolumn{3}{|c|}{2006} & \multicolumn{4}{|c|}{2007} & \multicolumn{2}{|c|}{2008} \\
\hline & & & Exeter & RCA & RCB & Exeter & Harrow & RCA & RCB & Env1 & Env2 \\
\hline Weed-free check & & & $100.0 \mathrm{a}$ & $100.0 \mathrm{a}$ & $100.0 \mathrm{a}$ & $100.0 \mathrm{a}$ & $100.0 \mathrm{a}$ & $100.0 \mathrm{a}$ & $100.0 \mathrm{a}$ & $100 \mathrm{a}$ & $100 \mathrm{a}$ \\
\hline Atrazine & PRE & 1000 & $99.7 \mathrm{ab}$ & $90.4 \mathrm{~b}$ & $92.7 \mathrm{~d}$ & $98.7 b$ & $99.4 \mathrm{a}$ & $95.4 \mathrm{~cd}$ & $89.3 d$ & $100 \mathrm{a}$ & $95 \mathrm{c}$ \\
\hline s-metolachlor/atrazine/benoxacor & PRE & 1080 & $93.4 \mathrm{~cd}$ & $71.4 \mathrm{c}$ & $91.3 \mathrm{~d}$ & $91.5 \mathrm{c}$ & $78.2 \mathrm{~b}$ & $76.4 \mathrm{e}$ & $79.3 \mathrm{e}$ & $98 \mathrm{a}$ & $46 \mathrm{~d}$ \\
\hline Isoxaflutole + atrazine & $\mathrm{PRE}+\mathrm{PRE}$ & $40+400$ & $99.7 \mathrm{ab}$ & $99.0 \mathrm{a}$ & $97.5 \mathrm{bc}$ & $100.0 \mathrm{a}$ & $98.1 \mathrm{a}$ & $96.8 \mathrm{c}$ & $76.6 \mathrm{e}$ & $100 \mathrm{a}$ & $97 \mathrm{bc}$ \\
\hline Dicamba/atrazine & PRE & 1000 & $99.7 \mathrm{ab}$ & $70.8 \mathrm{c}$ & $97.0 \mathrm{c}$ & $100.0 \mathrm{a}$ & $100.0 \mathrm{a}$ & $98.6 b$ & $99.0 \mathrm{~b}$ & $96 b$ & $100 \mathrm{a}$ \\
\hline Glyphosate & EPOST & 900 & $77.4 \mathrm{e}$ & $70.8 \mathrm{c}$ & $97.3 \mathrm{bc}$ & $91.4 \mathrm{c}$ & $87.6 \mathrm{~b}$ & $94.8 \mathrm{~d}$ & $95.8 \mathrm{c}$ & $97 b$ & $98 \mathrm{ab}$ \\
\hline Glyphosate & LPOST & 900 & $89.0 \mathrm{~d}$ & $78.1 \mathrm{bc}$ & $97.5 \mathrm{bc}$ & $91.4 \mathrm{c}$ & $100.0 \mathrm{a}$ & $96.8 \mathrm{c}$ & $98.8 b$ & $100 \mathrm{a}$ & $99 \mathrm{a}$ \\
\hline Dicamba/atrazine $f b$ glyphosate & PRE $f b$ LPOST & $1000 \mathrm{fb} 900$ & $100.0 \mathrm{a}$ & $100.0 \mathrm{a}$ & $98.8 \mathrm{~b}$ & $100.0 \mathrm{a}$ & $100.0 \mathrm{a}$ & $99.0 \mathrm{~b}$ & $99.0 \mathrm{~b}$ & $100 \mathrm{a}$ & $100 \mathrm{a}$ \\
\hline Atrazine $f b$ glyphosate & PRE $f b$ LPOST & $1000 \mathrm{fb} 900$ & $99.8 \mathrm{ab}$ & $99.7 \mathrm{a}$ & $97.8 \mathrm{bc}$ & $100.0 \mathrm{a}$ & $100.0 \mathrm{a}$ & $98.5 b$ & $99.0 \mathrm{~b}$ & $99 a$ & $100 \mathrm{a}$ \\
\hline $\begin{array}{l}\text { s-metolachlor/atrazine/benoxacor } \\
\mathrm{fb} \text { glyphosate }\end{array}$ & PRE $f b$ LPOST & $1080 \mathrm{fb} 900$ & $100.0 \mathrm{a}$ & $99.4 \mathrm{a}$ & $97.8 \mathrm{bc}$ & $100.0 \mathrm{a}$ & $100.0 \mathrm{a}$ & $98.5 b$ & $98.5 b$ & $100 \mathrm{a}$ & $100 \mathrm{a}$ \\
\hline $\begin{array}{l}\text { Isoxaflutole }+ \text { atrazine } f b \\
\text { glyphosate }\end{array}$ & $\begin{array}{l}\text { PRE + PRE } f b \\
\quad \text { LPOST }\end{array}$ & $\begin{array}{l}40+400 f b \\
900\end{array}$ & $100.0 \mathrm{a}$ & $99.9 \mathrm{a}$ & $98.5 \mathrm{bc}$ & $100.0 \mathrm{a}$ & $100.0 \mathrm{a}$ & $99.0 \mathrm{~b}$ & $99.0 \mathrm{~b}$ & $100 \mathrm{a}$ & $100 \mathrm{a}$ \\
\hline Glyphosate $f b$ glyphosate & $\begin{array}{l}\text { EPOST } f b \\
\text { LPOST }\end{array}$ & $900 f b 900$ & $97.2 \mathrm{bc}$ & $99.0 \mathrm{a}$ & $97.8 \mathrm{bc}$ & $100.0 \mathrm{a}$ & $100.0 \mathrm{a}$ & $98.4 \mathrm{~b}$ & $98.8 b$ & $100 \mathrm{a}$ & $99 \mathrm{a}$ \\
\hline \multicolumn{12}{|l|}{$\underline{\text { Contrasts }}^{\mathrm{b}}$} \\
\hline \multicolumn{3}{|l|}{ WF vs glyphosate EPOST } & $*$ & $*$ & $*$ & $*$ & $*$ & $*$ & $*$ & $*$ & NS \\
\hline \multicolumn{3}{|l|}{ WF vs glyphosate LPOST } & $*$ & $*$ & $*$ & $*$ & NS & $*$ & $*$ & $*$ & NS \\
\hline \multicolumn{3}{|c|}{ Glyphosate EPOST vs PRE $f b$ glyphosate LPOST } & $*$ & $*$ & NS & $*$ & $*$ & $*$ & $*$ & $*$ & NS \\
\hline \multicolumn{3}{|c|}{ Glyphosate LPOST vs PRE $f b$ glyphosate LPOST } & $*$ & $*$ & NS & * & NS & $*$ & $\mathrm{NS}$ & $*$ & NS \\
\hline \multicolumn{3}{|c|}{$\begin{array}{l}\text { Glyphosate EPOST } f b \text { glyphosate LPOST vs PRE } f b \\
\text { glyphosate LPOST }\end{array}$} & $*$ & $\mathrm{NS}$ & NS & NS & NS & $\mathrm{NS}$ & $\mathrm{NS}$ & $\mathrm{NS}$ & NS \\
\hline \multicolumn{3}{|c|}{$\begin{array}{l}\text { Glyphosate EPOST vs glyphosate EPOST } f b \text { glyphosate } \\
\text { LPOST }\end{array}$} & $*$ & $*$ & NS & $*$ & $*$ & $*$ & $*$ & $*$ & NS \\
\hline \multicolumn{3}{|c|}{$\begin{array}{l}\text { Glyphosate LPOST vs glyphosate EPOST } f b \text { glyphosate } \\
\text { LPOST }\end{array}$} & $*$ & $*$ & NS & $*$ & $\mathrm{NS}$ & $*$ & NS & $*$ & NS \\
\hline \multicolumn{3}{|c|}{ Glyphosate EPOST vs glyphosate LPOST } & * & NS & NS & NS & * & $*$ & * & NS & NS \\
\hline
\end{tabular}

${ }^{\mathrm{a}}$ Data were pooled by environment (location and year) when the interaction between environment and treatment was non-significant. Means are presented on the back-transformed scale. Means followed by the same letter within a column are not significantly different according to Fisher's Protected LSD (P < 0.05); ${ }^{\mathrm{b}}$ a priori orthogonal contrasts; * = significant $(\mathrm{P}<0.05)$; Abbreviations: CHEAL, common lambsquarters, PRE, preemergence; POST, postemergence; EPOST, early postemergence; LPOST, late postemergence; RCA, Ridgetown Site 1; RCB, Ridgetown Site 2; WF, weed-free; $f b$, followed by; Env1 = Exeter and Harrow 2008; Env2 = RCA and RCB 2008; NS, not significant. 
and RCA and in 2008 within Environment 1. Delaying glyphosate application improved common lambsquarters control by $12 \%, 12 \%, 2 \%$ and $3 \%$ at Exeter in 2006 and Harrow, RCA and RCB in 2007, respectively, compared to glyphosate applied EPOST (Table 4). A PRE herbicide $f b$ glyphosate only had higher common lambsquarters control compared to a sequential glyphosate application at Exeter in 2006, otherwise the two programs did not differ. Generally, sequential glyphosate application increased control by $3 \%-28 \%$ and $2 \%-21 \%$ compared to glyphosate applied alone EPOST or LPOST, respectively, depending on location. The benefits of sequential glyphosate applications on common lambsquarters have been previously reported in both corn and soybean where control was between $5 \%-9 \%$ and $4 \%-9 \%$ higher compared to a single application of glyphosate $[7,20]$. This makes sense because sequential in-crop applications of glyphosate offer a grower an opportunity to control weeds escaping an EPOST glyphosate application while late emerging weeds may have been too large at the time of application to be completely controlled by a LPOST glyphosate application $[5,16,18]$. However, under certain environmental conditions, a single application of glyphosate has been shown to provide adequate season long control of common lambsquarters eliminating the need for a sequential application [13].

\subsubsection{Velvetleaf}

Preemergence herbicides applied alone provided less than $75 \%$ control of velvetleaf within Environment 1 (Table 5). As expected, this is largely due to the inadequate control of velvetleaf with atrazine alone or S-me-

Table 5. Mean percent control of ABUTH in response to several weed management strategies 56 days after treatment at Ridgetown, ON from 2006 to $2008^{\mathrm{a}}$.

\begin{tabular}{|c|c|c|c|c|}
\hline \multirow{2}{*}{ Treatment } & \multirow{2}{*}{ Timing } & \multirow{2}{*}{$\begin{array}{c}\text { Rate } \\
\mathbf{g} \cdot \mathbf{a i} / \mathbf{a e} \cdot \mathbf{h} \mathbf{a}^{-1}\end{array}$} & \multicolumn{2}{|c|}{ Weed Control (\%) } \\
\hline & & & Env1 & Env2 \\
\hline Weed-free check & & & $100 \mathrm{a}$ & $100 \mathrm{a}$ \\
\hline Atrazine & PRE & 1000 & $1 \mathrm{~d}$ & $1 \mathrm{c}$ \\
\hline s-metolachlor/atrazine/benoxacor & PRE & 1080 & $1 \mathrm{~d}$ & $1 \mathrm{c}$ \\
\hline Isoxaflutole + atrazine & $\mathrm{PRE}+\mathrm{PRE}$ & $40+400$ & $74 \mathrm{~b}$ & $96 \mathrm{~b}$ \\
\hline Dicamba/atrazine & PRE & 1000 & $71 b$ & $99 \mathrm{a}$ \\
\hline Glyphosate & EPOST & 900 & $38 \mathrm{c}$ & $93 b$ \\
\hline Glyphosate & LPOST & 900 & $98 \mathrm{a}$ & $99 \mathrm{ab}$ \\
\hline Dicamba/atrazine $f b$ glyphosate & PRE $f b$ LPOST & $1000 f b 900$ & $70 \mathrm{~b}$ & $99 \mathrm{ab}$ \\
\hline Atrazine $f b$ glyphosate & PRE $f b$ LPOST & $1000 f b 900$ & $99 \mathrm{a}$ & $99 \mathrm{ab}$ \\
\hline s-metolachlor/atrazine/benoxacor $f b$ glyphosate & PRE $f b$ LPOST & $1080 f b 900$ & $99 \mathrm{a}$ & $100 \mathrm{a}$ \\
\hline Isoxaflutole + atrazine $f b$ glyphosate & PRE + PRE $f b$ LPOST & $40+400 f b 900$ & $99 \mathrm{a}$ & $100 \mathrm{a}$ \\
\hline Glyphosate $f b$ glyphosate & EPOST $f b$ LPOST & $900 f b 900$ & $98 \mathrm{a}$ & $99 \mathrm{ab}$ \\
\hline \multicolumn{5}{|l|}{ Contrasts ${ }^{\mathrm{b}}$} \\
\hline WF vs glyphosate EPOST & & & * & * \\
\hline WF vs glyphosate LPOST & & & NS & NS \\
\hline Glyphosate EPOST vs PRE $f b$ glyphosate LPOST & & & $*$ & NS \\
\hline Glyphosate LPOST vs PRE $f b$ glyphosate LPOST & & & NS & NS \\
\hline Glyphosate EPOST $f b$ glyphosate LPOST vs. PRE $f b$ glyphosate LPOST & & & NS & NS \\
\hline Glyphosate EPOST vs glyphosate EPOST $f b$ glyphosate LPOST & & & $*$ & NS \\
\hline Glyphosate LPOST vs glyphosate EPOST $f b$ glyphosate LPOST & & & NS & NS \\
\hline Glyphosate EPOST vs glyphosate LPOST & & & * & NS \\
\hline
\end{tabular}

${ }^{\mathrm{a}}$ Data were pooled by environment (location and year) when the interaction between environment and treatment was non-significant. Means are presented on the back-transformed scale. Means followed by the same letter within a column are not significantly different according to Fisher's Protected LSD (P < 0.05); ${ }^{\mathrm{b}}$ a priori orthogonal contrasts; ${ }^{*}=$ significant $(\mathrm{P}<0.05)$; Abbreviations: ABUTH, velvetleaf; PRE, preemergence; POST, postemergence; EPOST, early postemergence; LPOST, late postemergence; RCA, Ridgetown Site 1; RCB, Ridgetown Site 2; WF, weed-free; $f b$, followed by; Env1 = RCA 2006, RCB 2006, RCA; Env2 = RCA 2007 and RCA 2008; NS, not significant. 
tolachlor/atrazine/benoxacor [32]; highlighted by the $1 \%$ control within both environments. Glyphosate applied EPOST within Environment 1 also provided limited control of velvetleaf (38\%). Percent control was improved when glyphosate was preceded by a PRE herbicide. Sequential application of glyphosate or glyphosate applied LPOST both provided $60 \%$ better control of velvetleaf compared to a single glyphosate application EPOST in Environment1. This is supported by Gonzini et al. [20] who demonstrated that velvetleaf control was improved by $13 \%-22 \%$ and $17 \%-27 \%$ when PRE herbicides were followed by glyphosate or sequential applications of glyphosate were applied, respectively, compared to a single application of glyphosate.

\subsubsection{Annual Grasses}

Preemergence herbicides $f b$ glyphosate provided greater control of annual grasses than glyphosate applied EPOST at Exeter and RCB in 2006, Exeter and Harrow in 2007, and Harrow, RCA and RCB in 2008; or glyphosate applied LPOST at Exeter in 2007 (Table 6). Residual control from the PRE herbicide in addition to a LPOST cleanup with glyphosate likely contributed to the increased annual grass control. Annual grass control was higher when PRE herbicides were followed by glyphosate compared to sequential glyphosate applications at Exeter in 2008; however this relationship was reversed at Harrow in 2007 and 2008. The only non-glyphosate treatments expected to control annual grasses were $S$-metolachlor/atrazine/benoxacor and isoxaflutole + atrazine. Comparison of these treatments to a sequential glyphosate application showed no differences at Harrow $(2007,2008)$ and had higher control at Exeter in 2008. Control of annual grasses increased by $11 \%-70 \%$ and $6 \%-9 \%$ with sequential glyphosate applications compared to glyphosate applied alone EPOST or LPOST, respectively, depending on location (Table 6). As suggested previously, EPOST glyphosate applications alone may result in weed escapes and LPOST glyphosate applications alone may not control larger weeds. Application of glyphosate LPOST provided $11 \%$ - 64\% greater control of annual grasses compared to EPOST application; however, this response was location dependent. Gonzini et al. [20] found that in glyphosate-resistant soybean control of giant foxtail improved by $2 \%-15 \%$ with sequential applications of glyphosate POST or PRE herbicides followed by a POST application of glyphosate compared to a single-pass application of glyphosate POST.

\subsection{Crop Injury and Yield}

There was no visual crop injury at 7 and 28 DAT for all treatments in this study (data not shown). At most locations, PRE herbicides $f b$ glyphosate resulted in higher yields than when glyphosate was applied alone LPOST (Table 7). Nurse et al. [6] demonstrated that corn yield increased when a PRE herbicide (flufenacet + metribuzin) was $f b$ glyphosate compared to glyphosate alone. They attributed this response to early season weed control. In our study, sequential applications of glyphosate also had higher corn yield by up to $1.9 \mathrm{MT} \cdot \mathrm{ha}^{-1}$ compared to glyphosate applied LPOST at most locations. We attribute these higher yields to increased weed control. There was no difference in corn yield between glyphosate applied EPOST and sequential glyphosate applications; however, when glyphosate was applied EPOST yield increased by 1.7 MT $\cdot \mathrm{ha}^{-1}$ compared to glyphosate applied LPOST at Exeter in 2007. Greater control of common ragweed contributed to higher corn yield at this location with glyphosate applied EPOST. In contrast, glyphosate applied EPOST reduced yield by $1.1 \mathrm{MT} \cdot \mathrm{ha}^{-1}$ compared to glyphosate applied LPOST at RCB in 2008. Again, we attributed this to a decrease in weed control. At all other locations, there was no difference in corn yield between EPOST and LPOST glyphosate.

\subsection{Environmental Impact}

The type of application impacts the EIQ of a pesticide. Kovach et al. [23] considered that all POST herbicides have a greater risk due to plant surface persistence, which factors into the farm worker, consumer and environmental components of the EIQ calculation. The EIQ of PRE applied atrazine, metolachlor, and isoxaflutole (Table 8) were on average 8.8 lower than the same herbicide applied POST. With approximately an order of magnitude difference in the EIQ, it is critical to consider the type of application when evaluating the relative risk of weed control strategies.

The lowest EI of 6.0 was atrazine + isoxaflutole, due to the low application rate. In contrast, due to higher EIQ values, the highest EI of herbicide products applied alone was atrazine + dicamba at 24.6. Clearly, adding another active ingredient to the weed management strategy will increase the EI, but for all treatments other than atrazine + isoxaflutole, adding glyphosate less than doubles the EI. For atrazine + isoxaflutole the addition of glyphosate more than triples the EI.

Sequential glyphosate applications had a higher EI than all products applied alone and atrazine + isoxaflutole $f b$ glyphosate. The EI of applying glyphosate twice was equivalent to applying atrazine + dicamba alone, atrazine $f b$ glyphosate, atrazine + metolachlor $f b$ glyphosate and atrazine + isoxaflutole $f b$ glyphosate. Thus, the addition of the aforementioned PRE herbicides to 
Table 6. Mean percent control of annual grasses in response to several weed management strategies 56 days after treatment at Exeter and Ridgetown, ON from 2006 to 2008 and Harrow, ON from 2007 to 2008 .

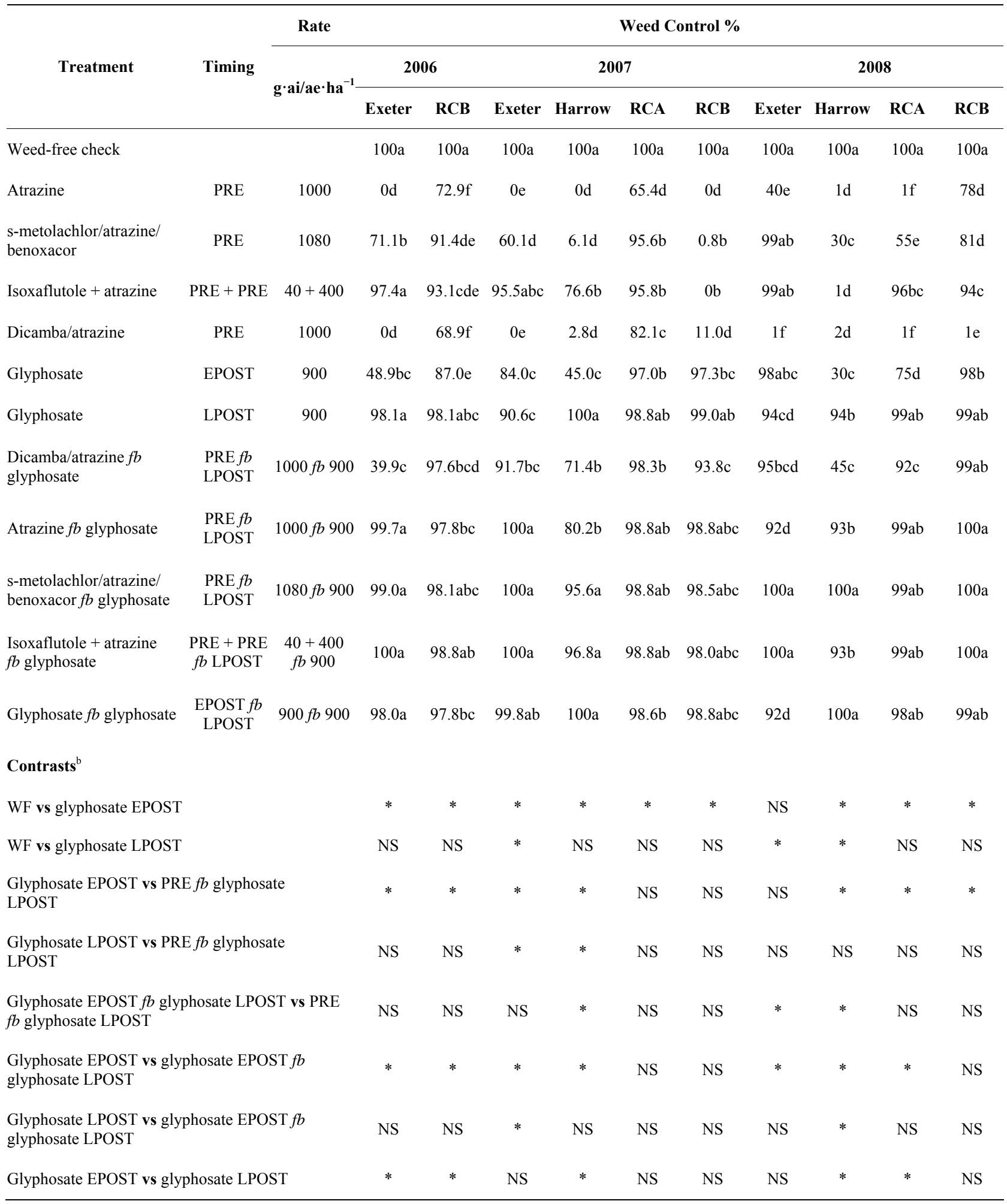

${ }^{a}$ Data were pooled by environment (location and year) when the interaction between environment and treatment was non-significant. Means are presented on the back-transformed scale. Means followed by the same letter within a column are not significantly different according to Fisher's Protected LSD (P $<0.05)$. ${ }^{\mathrm{b}} a$ priori orthogonal contrasts. $*=$ significant $(\mathrm{P}<0.05)$; Abbreviations: PRE, preemergence; POST, postemergence; EPOST, early postemergence; LPOST, late postemergence; WF, weed-free; $f b$, followed by; RCA, Ridgetown Site 1; RCB, Ridgetown Site 2; NS, not significant. 
Table 7. Mean corn yield in response to several weed management strategies at Exeter and Ridgetown, ON from 2006 to 2008 and Harrow, ON from 2007 to $2008^{\mathrm{a}}$.

\begin{tabular}{|c|c|c|c|c|c|c|c|c|c|c|c|}
\hline \multirow{3}{*}{ Treatment } & \multicolumn{11}{|c|}{ Yield MT $\cdot \mathrm{ha}^{-1}$} \\
\hline & \multicolumn{3}{|c|}{2006} & \multicolumn{4}{|c|}{2007} & \multicolumn{4}{|c|}{2008} \\
\hline & RCA & RCB & Exeter & RCA & RCB & Exeter & Harrow & RCA & RCB & Exeter & Harrow \\
\hline Weedy check & $2.0 \mathrm{~d}$ & $1.4 \mathrm{~d}$ & $7.1 \mathrm{c}$ & $5.0 \mathrm{~d}$ & $0.7 \mathrm{f}$ & $5.0 \mathrm{e}$ & $5.0 \mathrm{~d}$ & $3.7 \mathrm{e}$ & $8.5 \mathrm{~d}$ & $6.6 \mathrm{~b}$ & $6.3 \mathrm{~d}$ \\
\hline Weed-free check & $12.8 \mathrm{a}$ & $10.0 \mathrm{a}$ & $11.8 \mathrm{ab}$ & $12.7 \mathrm{a}$ & $12.0 \mathrm{a}$ & $11.0 \mathrm{ab}$ & $12.6 \mathrm{a}$ & $11.2 \mathrm{a}$ & $15.0 \mathrm{a}$ & $12.4 \mathrm{a}$ & $16.7 \mathrm{ab}$ \\
\hline Atrazine & $8.4 \mathrm{~b}$ & $4.2 \mathrm{c}$ & $11.0 \mathrm{ab}$ & $9.5 \mathrm{c}$ & $4.5 \mathrm{~d}$ & $10.0 \mathrm{bcd}$ & $10.1 \mathrm{c}$ & $8.1 \mathrm{~cd}$ & $14.0 \mathrm{ab}$ & $11.7 \mathrm{a}$ & $12.8 \mathrm{c}$ \\
\hline s-metolachlor/atrazine/benoxacor & $5.9 \mathrm{c}$ & $4.6 \mathrm{c}$ & $11.0 \mathrm{ab}$ & $9.8 \mathrm{c}$ & $3.0 \mathrm{e}$ & $9.7 \mathrm{~cd}$ & $10.9 \mathrm{c}$ & $7.5 \mathrm{~d}$ & $12.1 \mathrm{c}$ & $12.0 \mathrm{a}$ & $15.7 b$ \\
\hline Isoxaflutole + atrazine & $12.6 \mathrm{a}$ & $8.6 \mathrm{ab}$ & $11.8 \mathrm{ab}$ & $12.1 \mathrm{a}$ & $7.3 \mathrm{c}$ & $10.8 \mathrm{abc}$ & $12.6 \mathrm{ab}$ & $10.6 \mathrm{ab}$ & $13.4 \mathrm{~b}$ & $12.8 \mathrm{a}$ & $15.6 b$ \\
\hline Dicamba/atrazine & $12.6 \mathrm{a}$ & $8.4 \mathrm{~b}$ & $11.3 \mathrm{ab}$ & $11.2 \mathrm{~b}$ & $10.4 \mathrm{~b}$ & $10.0 \mathrm{bcd}$ & $9.4 \mathrm{c}$ & $9.1 \mathrm{c}$ & $13.7 \mathrm{~b}$ & $12.9 \mathrm{a}$ & $11.7 \mathrm{c}$ \\
\hline Glyphosate & $12.6 \mathrm{a}$ & $8.7 \mathrm{ab}$ & $11.3 \mathrm{ab}$ & $11.8 \mathrm{ab}$ & $11.7 \mathrm{ab}$ & $11.1 \mathrm{ab}$ & $12.6 \mathrm{ab}$ & $10.6 \mathrm{ab}$ & $13.8 \mathrm{~b}$ & $12.1 \mathrm{a}$ & $15.8 b$ \\
\hline Glyphosate & $12.3 \mathrm{a}$ & $8.1 \mathrm{~b}$ & $10.7 \mathrm{~b}$ & $11.4 \mathrm{ab}$ & $10.7 \mathrm{~b}$ & $9.4 \mathrm{~d}$ & $11.1 \mathrm{bc}$ & $9.9 \mathrm{bc}$ & $14.9 \mathrm{a}$ & $11.6 \mathrm{a}$ & $14.5 b$ \\
\hline Dicamba/atrazine $f b$ glyphosate & $12.6 \mathrm{a}$ & $8.5 \mathrm{~b}$ & $12.0 \mathrm{a}$ & $12.5 \mathrm{ab}$ & $12.8 \mathrm{a}$ & $10.5 \mathrm{abcd}$ & $12.6 \mathrm{ab}$ & $10.7 \mathrm{ab}$ & $14.4 \mathrm{a}$ & $11.9 \mathrm{a}$ & $15.9 \mathrm{~b}$ \\
\hline Atrazine $f b$ glyphosate & $13.0 \mathrm{a}$ & $8.7 \mathrm{ab}$ & $11.9 \mathrm{ab}$ & $12.0 \mathrm{ab}$ & $11.9 \mathrm{ab}$ & $10.5 \mathrm{abcd}$ & $13.7 \mathrm{a}$ & $11.1 \mathrm{a}$ & $13.9 \mathrm{~b}$ & $12.8 \mathrm{a}$ & $16.2 \mathrm{ab}$ \\
\hline $\begin{array}{l}\text { s-metolachlor/atrazine/benoxacor } f b \\
\text { glyphosate }\end{array}$ & $13.7 \mathrm{a}$ & $9.0 \mathrm{ab}$ & $12.1 \mathrm{a}$ & $11.9 \mathrm{ab}$ & $11.7 \mathrm{ab}$ & $11.1 \mathrm{ab}$ & $13.4 \mathrm{a}$ & $10.1 \mathrm{abc}$ & $13.8 \mathrm{~b}$ & $12.4 \mathrm{a}$ & $17.6 \mathrm{a}$ \\
\hline Isoxaflutole + atrazine $f b$ glyphosate & $13.6 \mathrm{a}$ & $8.8 \mathrm{ab}$ & $11.9 \mathrm{ab}$ & $12.2 \mathrm{ab}$ & $12.4 \mathrm{a}$ & $11.3 \mathrm{a}$ & $12.8 \mathrm{ab}$ & $10.5 \mathrm{ab}$ & $13.8 \mathrm{~b}$ & $12.1 \mathrm{a}$ & $16.5 \mathrm{ab}$ \\
\hline Glyphosate $f b$ glyphosate & $13.9 \mathrm{a}$ & $9.5 \mathrm{ab}$ & $12.2 \mathrm{a}$ & $12.1 \mathrm{ab}$ & $12.6 \mathrm{a}$ & $11.2 \mathrm{ab}$ & $13.2 \mathrm{ab}$ & $10.7 \mathrm{ab}$ & $13.8 \mathrm{~b}$ & $12.5 \mathrm{a}$ & $16.1 \mathrm{ab}$ \\
\hline $\mathrm{LSD}_{0.05}$ & 2.02 & 1.49 & 1.21 & 1.51 & 1.23 & 1.28 & 2.20 & 1.10 & 1.00 & 3.21 & 1.50 \\
\hline \multicolumn{12}{|l|}{$\underline{\text { Contrasts }}^{\mathrm{b}}$} \\
\hline WF vs glyphosate EPOST & NS & $*$ & NS & NS & NS & NS & NS & NS & $*$ & NS & NS \\
\hline WF vs glyphosate LPOST & NS & $*$ & $*$ & NS & $*$ & $*$ & $*$ & $*$ & NS & NS & NS \\
\hline $\begin{array}{l}\text { Glyphosate EPOST vs PRE } f b \\
\text { glyphosate LPOST }\end{array}$ & NS & NS & NS & NS & NS & NS & NS & NS & NS & NS & NS \\
\hline $\begin{array}{l}\text { Glyphosate LPOST vs PRE } f b \\
\text { glyphosate LPOST }\end{array}$ & NS & $*$ & $*$ & NS & $*$ & $*$ & $*$ & $*$ & $*$ & NS & NS \\
\hline $\begin{array}{l}\text { Glyphosate EPOST } f b \text { glyphosate } \\
\text { LPOST vs PRE } f b \text { glyphosate LPOST }\end{array}$ & NS & NS & NS & NS & NS & NS & NS & NS & NS & NS & NS \\
\hline $\begin{array}{l}\text { Glyphosate EPOST vs glyphosate } \\
\text { EPOST } f b \text { glyphosate LPOST }\end{array}$ & NS & NS & NS & NS & NS & NS & NS & NS & NS & NS & NS \\
\hline $\begin{array}{l}\text { Glyphosate LPOST vs glyphosate } \\
\text { EPOST } f b \text { glyphosate LPOST }\end{array}$ & $*$ & $*$ & $*$ & NS & $*$ & $*$ & NS & NS & $*$ & NS & NS \\
\hline $\begin{array}{l}\text { Glyphosate EPOST vs glyphosate } \\
\text { LPOST }\end{array}$ & NS & NS & NS & NS & NS & $*$ & NS & NS & $*$ & NS & NS \\
\hline
\end{tabular}


Table 8. Environmental impact quotient (EIQ) and environmental impact (EI) of weed management strategies used at Exeter and Ridgetown, ON from 2006 to 2008 and Harrow, ON from 2007 to 2008.

\begin{tabular}{|c|c|c|c|c|}
\hline Active ingredient(s) & Timing & Individual EIQ values $^{\mathrm{a}}$ & Product Rate $\mathbf{g} \cdot \mathbf{a i} / \mathbf{a e} \cdot \mathbf{h a}^{-1}$ & $\mathbf{E I}^{\mathbf{b}}$ \\
\hline Atrazine & PRE & 13.6 & 1000 & 13.6 \\
\hline s-metolachlor/atrazine/benoxacor & PRE & $14.2 / 13.6$ & 1080 & 15.0 \\
\hline Atrazine + isoxaflutole & PRE & $13.6 / 13.3$ & 440 & 6.0 \\
\hline Dicamba/atrazine & PRE & $22.9 / 28.0$ & 1000 & 24.6 \\
\hline Glyphosate & EPOST & 15.3 & 900 & 13.8 \\
\hline Glyphosate & LPOST & & 900 & 13.8 \\
\hline Dicamba/atrazine $f b$ glyphosate & PRE $f b$ LPOST & & $1000 f b 900$ & 38.4 \\
\hline Atrazine $f b$ glyphosate & PRE $f b$ LPOST & & $1000 f b 900$ & 27.4 \\
\hline$s$-metolachlor/atrazine/benoxacor $f b$ glyphosate & PRE $f b$ LPOST & & $1080 f b 900$ & 28.8 \\
\hline Atrazine + isoxaflutole $f b$ glyphosate & PRE $f b$ LPOST & & $440 f b 900$ & 19.8 \\
\hline Glyphosate $f b$ glyphosate & EPOST $f b$ LPOST & & 1800 & 27.5 \\
\hline
\end{tabular}

${ }^{a}$ EIQ values for each a.i. obtained from Kovach et al. (1999), except for atrazine, metolachlor, and isoxaflutole which were calculated according to formula developed by Kovach et al. (1992) using PRE vs POST application; ${ }^{b}$ EI values for products with more than one a.i. were obtained by summing the relative proportion of each a.i.; Abbreviations: EPOST, early postemergence; LPOST, late postemergence; $f b$, followed by; EIQ, environmental impact quotient; EI, environmental impact.

glyphosate-resistant corn production is strongly recommended based on equivalent environmental risk as well as resistance management.

\subsection{Profitability Analysis}

The results of the profitability analysis indicate that across all locations and years no significant differences in profit margins exist between the following treatments: isoxaflutole + atrazine, glyphosate EPOST, glyphosate LPOST, dicamba/atrazine followed by $(f b)$ glyphosate, atrazine $f b$ glyphosate, $S$-metolachlor/atrazine/benoxacor $f b$ glyphosate, isoxaflutole + atrazine $f b$ glyphosate and sequential applications of glyphosate (Table 9). Aside from the weedy check treatment, the lowest profit margins were found in the atrazine and S-metolachlor/ atrazine/benoxacor treatments. Other than the weedy check, relatively few significant differences existed between treatments within each location and within each year. At both Ridgetown locations, profit margins across all three years only were reduced for the atrazine and $S$-metolachlor/atrazine/benoxacor treatments compared to almost all other treatments. At Exeter, profit margins for the $S$-metolachlor/atrazine/benoxacor treatment were lower than the profit margins for dicamba/atrazine, glyphosate EPOST and sequential applications of glyphosate. At Harrow, only the atrazine and dicamba/atrazine treatments were significantly lower in profit margins than most of the other treatments. Similarly, other than the weedy check, there are few differences in profit margins between treatments found within each year. In 2006 and 2007, atrazine and S-metolachlor/atrazine/benoxacor have reduced profit margins compared to all other treatments. In addition, in 2007 isoxaflutole + atrazine had reduced profit margins compared to several other treatments (Table 9). In 2008, there are no differences in profit margins between any of the herbicide treatments.

Relatively little change in these results can be found when examining profit margins within each location-year (Table 10), as the significant differences that exist are consistent for the most part with the results discussed above. Among the 11 location-years, treatments that are found to have recurring lower profit margins compared to other treatments include the atrazine, S-metolachlor/ atrazine/benoxacor, dicamba/atrazine and glyphosate LPOST treatments. Overall, profit margins tend to be somewhat higher for treatments that include glyphosate applications.

\section{Conclusions}

In summary, PRE herbicides $f b$ glyphosate LPOST and sequential glyphosate applications provided greater control of annual broadleaf weeds and annual grasses compared to single glyphosate applications. This is due to control of weed escapes through residual control of PRE herbicides and/or the control provided by a second glyphosate application LPOST. Generally, weed control with glyphosate applied LPOST was greater than weed control with glyphosate applied EPOST; however, this 
Table 9. Profit margins over weed control costs by treatment for corn from 2006-2008 $\left(\mathrm{Cdn}^{\mathrm{h}} \mathrm{ha}^{-1}\right){ }^{\mathrm{a}}$

\begin{tabular}{|c|c|c|c|c|c|c|c|c|}
\hline \multirow{2}{*}{ Treatment } & \multirow{2}{*}{ All locations } & RCA & RCB & Exeter & Harrow & \multirow{2}{*}{2006} & \multirow{2}{*}{2007} & \multirow{2}{*}{2008} \\
\hline & & $(2006-2008)$ & (2006-2008) & $(2006-2008)$ & (2006-2008) & & & \\
\hline Weedy check & $757.75 d$ & $543.18 \mathrm{c}$ & $543.99 \mathrm{~d}$ & $1114.82 \mathrm{c}$ & $864.61 d$ & $536.91 \mathrm{c}$ & $720.47 \mathrm{e}$ & $960.65 b$ \\
\hline Atrazine & $1412.22 \mathrm{c}$ & $1289.26 b$ & $1125.34 b c$ & $1621.11 \mathrm{ab}$ & $1713.65 \mathrm{bc}$ & $1169.70 \mathrm{~b}$ & $1259.09 \mathrm{~d}$ & $1747.24 \mathrm{a}$ \\
\hline s-metolachlor/atrazine/benoxacor & $1351.99 \mathrm{c}$ & $1131.82 \mathrm{~b}$ & $957.38 \mathrm{c}$ & $1541.53 b$ & $1989.87 \mathrm{ab}$ & $1049.47 \mathrm{~b}$ & $1167.81 \mathrm{~d}$ & $1763.07 \mathrm{a}$ \\
\hline Isoxaflutole + atrazine & $1708.07 \mathrm{ab}$ & $1748.63 \mathrm{a}$ & $1440.23 \mathrm{ab}$ & $1674.68 \mathrm{ab}$ & $2099.08 \mathrm{a}$ & $1630.41 \mathrm{a}$ & $1525.66 \mathrm{c}$ & $1948.72 \mathrm{a}$ \\
\hline Dicamba/atrazine & $1640.18 b$ & $1630.19 \mathrm{a}$ & $1608.95 \mathrm{a}$ & $1731.40 \mathrm{a}$ & $1565.19 \mathrm{c}$ & $1599.44 \mathrm{a}$ & $1543.63 b c$ & $1767.29 \mathrm{a}$ \\
\hline Glyphosate EPOST & $1802.30 \mathrm{ab}$ & $1743.29 \mathrm{a}$ & $1700.10 \mathrm{a}$ & $1740.17 \mathrm{a}$ & $2137.29 \mathrm{a}$ & $1619.93 a$ & $1780.93 a$ & $1960.43 a$ \\
\hline Glyphosate LPOST & $1707.24 \mathrm{ab}$ & $1671.00 \mathrm{a}$ & $1678.37 \mathrm{a}$ & $1631.36 \mathrm{ab}$ & $1918.74 \mathrm{abc}$ & $1542.99 \mathrm{a}$ & $1630.55 \mathrm{abc}$ & $1907.12 \mathrm{a}$ \\
\hline Dicamba/atrazine $f b$ glyphosate & $1772.99 \mathrm{ab}$ & $1733.26 \mathrm{a}$ & $1730.80 \mathrm{a}$ & $1644.70 \mathrm{ab}$ & $2088.29 \mathrm{ab}$ & $1595.85 \mathrm{a}$ & $1744.40 \mathrm{abc}$ & $1934.42 \mathrm{a}$ \\
\hline Atrazine $f b$ glyphosate & $1809.47 \mathrm{a}$ & $1764.77 \mathrm{a}$ & $1683.67 \mathrm{a}$ & $1715.58 \mathrm{ab}$ & $2206.08 \mathrm{a}$ & $1637.21 \mathrm{a}$ & $1760.95 \mathrm{ab}$ & $1987.19 a$ \\
\hline $\begin{array}{l}\text { s-metolachlor/atrazine/benoxacor } f b \\
\text { glyphosate }\end{array}$ & $1806.33 \mathrm{a}$ & $1730.03 \mathrm{a}$ & $1670.69 \mathrm{a}$ & $1703.11 \mathrm{ab}$ & $2279.08 \mathrm{a}$ & $1685.68 \mathrm{a}$ & $1734.02 \mathrm{abc}$ & $1969.12 \mathrm{a}$ \\
\hline Isoxaflutole + atrazine $f b$ glyphosate & $1782.02 \mathrm{ab}$ & $1749.30 \mathrm{a}$ & $1687.47 \mathrm{a}$ & $1672.54 \mathrm{ab}$ & $2137.15 \mathrm{a}$ & $1648.68 \mathrm{a}$ & $1738.68 \mathrm{abc}$ & $1925.37 \mathrm{a}$ \\
\hline Glyphosate $f b$ glyphosate & $1830.74 \mathrm{a}$ & $1787.24 \mathrm{a}$ & $1747.29 \mathrm{a}$ & $1740.72 \mathrm{a}$ & $2156.20 \mathrm{a}$ & $1729.58 \mathrm{a}$ & $1793.85 \mathrm{a}$ & $1943.50 \mathrm{a}$ \\
\hline
\end{tabular}

${ }^{a}$ Means within columns that are followed by the same letter are not significantly different from each other $(\mathrm{P}<0.05)$; Abbreviations: EPOST, early postemergence; LPOST, late postemergence; $f b$, followed by; RCA, Ridgetown Site 1; RCB, Ridgetown Site 2.

Table 10. Profit margins over weed control costs by treatment for corn, by location-year $\left(\mathrm{Cdn}^{\mathrm{S} \mathrm{ha}}{ }^{-1}\right)$. $^{\mathrm{a}}$

\begin{tabular}{|c|c|c|c|c|c|c|c|c|c|c|c|}
\hline Treatment & $\begin{array}{l}\text { RCA } \\
2006\end{array}$ & $\begin{array}{l}\text { RCB } \\
2006\end{array}$ & $\begin{array}{c}\text { Exeter } \\
2006\end{array}$ & $\begin{array}{l}\text { RCA } \\
2007\end{array}$ & $\begin{array}{l}\text { RCB } \\
2007\end{array}$ & $\begin{array}{c}\text { Exeter } \\
2007\end{array}$ & $\begin{array}{c}\text { Harrow } \\
2007\end{array}$ & $\begin{array}{l}\text { RCA } \\
2008\end{array}$ & $\begin{array}{l}\text { RCB } \\
2008\end{array}$ & $\begin{array}{c}\text { Exeter } \\
2008\end{array}$ & $\begin{array}{l}\text { Harrow } \\
2008\end{array}$ \\
\hline Weedy check & $309.03 \mathrm{~d}$ & $220.85 c$ & $1080.86 \mathrm{~b}$ & $759.72 d$ & $105.20 \mathrm{~g}$ & $1257.78 \mathrm{bc}$ & $759.17 \mathrm{e}$ & $560.80 \mathrm{f}$ & $1305.91 \mathrm{c}$ & $1005.83 \mathrm{c}$ & $970.05 \mathrm{~d}$ \\
\hline Atrazine & $1246.26 b$ & $609.40 \mathrm{~b}$ & $1653.45 \mathrm{a}$ & $1420.30 \mathrm{c}$ & $659.38 \mathrm{e}$ & 1451.99abc & $1504.68 \mathrm{~cd}$ & $1201.23 \mathrm{de}$ & $2107.22 \mathrm{ab}$ & $1757.88 \mathrm{ab}$ & $1922.61 b c$ \\
\hline $\begin{array}{c}s \text {-metolachlor/ } \\
\text { atrazine/benoxacor }\end{array}$ & $853.03 \mathrm{c}$ & $653.65 b$ & $1641.72 \mathrm{a}$ & $1449.31 b c$ & $408.05 \mathrm{f}$ & $1195.94 \mathrm{c}$ & $1617.95 \mathrm{bcd}$ & $1093.12 \mathrm{e}$ & $1810.42 b$ & $1786.94 \mathrm{ab}$ & $2361.78 \mathrm{ab}$ \\
\hline $\begin{array}{l}\text { Isoxaflutole }+ \\
\text { atrazine }\end{array}$ & $1875.54 \mathrm{a}$ & $1260.92 \mathrm{a}$ & $1754.77 \mathrm{a}$ & $1799.10 \mathrm{a}$ & $1060.29 \mathrm{~d}$ & $1368.47 \mathrm{abc}$ & $1874.78 \mathrm{ab}$ & $1571.23 \mathrm{ab}$ & $1999.48 \mathrm{ab}$ & $1900.81 \mathrm{ab}$ & $2323.38 \mathrm{ab}$ \\
\hline Dicamba/atrazine & $1878.06 \mathrm{a}$ & $1241.97 \mathrm{a}$ & $1678.30 \mathrm{a}$ & $1664.68 \mathrm{abc}$ & $1537.75 \mathrm{c}$ & $1589.01 \mathrm{ab}$ & $1383.06 \mathrm{~d}$ & $1347.83 \mathrm{~cd}$ & $2047.12 \mathrm{ab}$ & $1926.91 \mathrm{a}$ & $1747.31 \mathrm{c}$ \\
\hline Glyphosate EPOST & $1882.32 \mathrm{a}$ & $1286.49 \mathrm{a}$ & $1690.99 a$ & $1765.79 \mathrm{a}$ & $1744.34 \mathrm{abc}$ & $1721.71 \mathrm{a}$ & $1891.90 \mathrm{ab}$ & $1581.77 \mathrm{ab}$ & $2069.48 \mathrm{ab}$ & $1807.81 \mathrm{ab}$ & $2382.68 \mathrm{ab}$ \\
\hline Glyphosate LPOST & $1834.39 \mathrm{a}$ & $1191.40 \mathrm{a}$ & $1603.19 \mathrm{a}$ & $1707.35 \mathrm{ab}$ & $1604.13 b c$ & 1548.89abc & $1661.85 \mathrm{bcd}$ & $1471.25 \mathrm{abc}$ & $2239.57 \mathrm{a}$ & $1742.00 \mathrm{~b}$ & $2175.63 \mathrm{abc}$ \\
\hline $\begin{array}{c}\text { Dicamba/atrazine } \\
f b \text { glyphosate }\end{array}$ & $1834.08 \mathrm{a}$ & $1206.81 \mathrm{a}$ & $1746.66 \mathrm{a}$ & $1820.32 \mathrm{a}$ & $1867.73 \mathrm{a}$ & $1460.07 \mathrm{abc}$ & $1829.48 \mathrm{abc}$ & $1545.37 \mathrm{ab}$ & $2117.86 a$ & $1727.37 \mathrm{~b}$ & $2347.10 \mathrm{ab}$ \\
\hline $\begin{array}{l}\text { Atrazine } f b \\
\text { glyphosate }\end{array}$ & $1910.84 a$ & $1257.12 \mathrm{a}$ & $1743.67 \mathrm{a}$ & $1766.26 \mathrm{a}$ & $1736.89 \mathrm{abc}$ & $1528.20 \mathrm{abc}$ & $2012.45 a$ & $1617.20 \mathrm{a}$ & $2056.99 \mathrm{ab}$ & $1874.88 \mathrm{ab}$ & $2399.70 \mathrm{ab}$ \\
\hline $\begin{array}{c}s \text {-metolachlor/ } \\
\text { atrazine/benoxacor } \\
\text { fb glyphosate }\end{array}$ & $2005.32 \mathrm{a}$ & $1289.86 a$ & $1761.85 \mathrm{a}$ & $1733.03 \mathrm{ab}$ & $1703.40 \mathrm{abc}$ & $1546.49 \mathrm{abc}$ & $1953.18 \mathrm{ab}$ & $1451.72 b c$ & $2018.80 \mathrm{ab}$ & $1800.97 \mathrm{ab}$ & $2604.99 \mathrm{a}$ \\
\hline $\begin{array}{c}\text { Isoxaflutole }+ \\
\text { atrazine } f b \\
\text { glyphosate }\end{array}$ & $1978.42 \mathrm{a}$ & $1250.31 \mathrm{a}$ & $1717.31 \mathrm{a}$ & $1762.15 \mathrm{a}$ & $1795.44 \mathrm{ab}$ & $1543.70 \mathrm{abc}$ & $1853.42 \mathrm{ab}$ & $1507.33 \mathrm{abc}$ & $2016.67 \mathrm{ab}$ & $1756.60 \mathrm{ab}$ & $2420.88 \mathrm{ab}$ \\
\hline $\begin{array}{l}\text { Glyphosate } f b \\
\text { glyphosate }\end{array}$ & $2040.41 \mathrm{a}$ & $1372.11 \mathrm{a}$ & $1776.23 \mathrm{a}$ & $1763.34 \mathrm{a}$ & $1847.29 \mathrm{a}$ & $1625.20 \mathrm{ab}$ & $1939.57 \mathrm{ab}$ & $1557.97 \mathrm{ab}$ & $2022.48 \mathrm{ab}$ & $1820.73 \mathrm{ab}$ & $2372.83 \mathrm{ab}$ \\
\hline
\end{tabular}

${ }^{\mathrm{a}}$ Means within columns that are followed by the same letter are not significantly different from each other $(\mathrm{P}<0.05)$. Abbreviations: EPOST, early postemergence; LPOST, late postemergence; $f b$, followed by; RCA, Ridgetown Site 1; RCB, Ridgetown Site 2. 
generally did not translate into a yield benefit and often resulted in yield losses due to prolonged early season weed competition. A yield benefit was found with both sequential herbicide programs compared to a single application of glyphosate LPOST. However, corn yield did not differ between the sequential herbicide programs and glyphosate applied EPOST.

Based on the EIQ, the sequential herbicide programs had a greater environmental impact than one-pass herbicides programs, except dicamba/atrazine alone, which was equivalent to several two-pass treatments. Isoxaflutole/atrazine $f b$ glyphosate had the lowest environmental impact of the sequential herbicide programs evaluated. Overall, profit margins were moderately higher for treatments that included a glyphosate application (glyphosate alone or applied LPOST following a PRE herbicide). While glyphosate applied EPOST may appear to achieve the same management goals, the impact of reduced weed control over time could negatively affect future profit margins. Overall, these data showed that glyphosate $f b$ glyphosate was the most efficacious and profitable treatment; however, for the purposes of glyphosate stewardship, and reduced environmental impact, isoxaflutole/atrazine $f b$ glyphosate is also recommended as a profitable weed management system.

\section{Acknowledgements}

The authors would like to acknowledge the technical assistance of Lynette Brown, Todd Cowan, Elaine Lepp, and Christy Shropshire.

\section{REFERENCES}

[1] G. R. Armel, H. P. Wilson, R. J. Richardson and T. E. Hines, "Mesotrione, Acetochlor, and Atrazine for Weed Management in Corn (Zea mays)," Weed Technology, Vol. 17, No. 2, 2003, pp. 284-290. doi:10.1614/0890-037X(2003)017[0284:MAAAFW]2.0. $\underline{\mathrm{CO} ; 2}$

[2] B. G. Young, "Changes in Herbicide Use Patterns and Production Practices Resulting from Glyphosate-Resistant Crops," Weed Technology, Vol. 20, No. 2, 2006, pp. 301-307. doi:10.1614/WT-04-189.1

[3] G. R. W. Nice, F. Whitford, B. Johnson and C. Janssen, "Assessing the Impact of Educating Growers about Proper Use of Atrazine in Pesticide Applicator Recertification Programs," Weed Technology, Vol. 22, No. 2, 2008, pp. 326-330. doi:10.1614/WT-07-146.1

[4] C. J. Swanton, R. H. Gulden and K. Chandler, "A Rationale for Atrazine Stewardship in Corn," Weed Science, Vol. 55, No. 1, 2007, pp. 75-81. doi:10.1614/WS-06-104.1

[5] J. W. Barnes and L. R. Oliver, "Cloransulam Antagonizes Annual Grass Control with Aryloxyphenoxypropionate Graminicides but Not Cyclohexanediones," Weed Tech- nology, Vol. 18, 2004, pp. 763-772. doi:10.1614/WT-03-181R

[6] R. E. Nurse, C. J. Swanton., F. J. Tardif and P. H. Sikkema, "Weed Control and Yield Are Improved When Glyphosate Is Preceded by a Residual Herbicide in Glyphosate-Tolerant Maize (Zea mays)," Crop Protection, Vol. 25, No. 11, 2006, pp. 1174-1179. doi:10.1016/j.cropro.2006.02.015

[7] C. L. Stewart, R. E. Nurse, A. S. Hamill and P. H. Sikkema, "Precipitation Influences Pre- and Post-Emergence Herbicide Efficacy in Corn," American Journal of Plant Science, Vol. 3, No. 9, 2012, pp. 1193-1204. doi:10.4236/ajps.2012.39145

[8] S. O. Duke, "Taking Stock of Herbicide-Resistant Crops Ten Years after Introduction," Pest Management Science, Vol. 61, No. 3, 2005, pp. 211-218. doi:10.1002/ps.1024

[9] I. Heap, "The International Survey of Herbicide Resistant Weeds," 2012. http://www.weedscience.org

[10] R. E. Jones and R. W. Medd, "Economic Thresholds and the Case for Longer Term Approaches to Population Management of Weeds," Weed Technology, Vol. 14, No. 2, 2000, pp. 337-350. doi:10.1614/0890-037X(2000)014[0337:ETATCF]2.0.C $\mathrm{O} ; 2$

[11] J. A. Bond and J. L. Griffin, "Weed Control in Corn (Zea mays) with an Imazethapyr Plus Imazapyr Prepackaged Mixture," Weed Technology, Vol. 19, No. 4, 2005, pp. 992-998. doi:10.1614/WT-04-267R1.1

[12] B. C. Johnson, B. G. Young and J. L. Matthews, "Effect of Postemergence Application Rate and Timing of Mesotrione on Corn (Zea mays) Response and Weed Control," Weed Technology, Vol. 16, No. 2, 2002, pp. 414-420. doi:10.1614/0890-037X(2002)016[0414:EOPARA]2.0.C $\underline{\mathrm{O} ; 2}$

[13] C. L. Stewart, R. E. Nurse, A. S. Hamill and P. H. Sikkema, "Environment and Soil Conditions Influence Preand Post-Emergence Herbicide Efficacy in Soybean," Weed Technology, Vol. 24, No. 3, 2010, pp. 234-243. doi:10.1614/WT-09-009.1

[14] C. J. Swanton, A. Shrestha, K. Chandler and W. Deen, "An Economic Assessment of Weed Control Strategies in No-Till Glyphosate-Resistant Soybean (Glycine max)," Weed Technology, Vol. 14, No. 4, 2000, pp. 755-763. doi:10.1614/0890-037X(2000)014[0755:AEAOWC]2.0.C $\underline{\mathrm{O} ; 2}$

[15] B. Hartzler, "Is One-Pass Weed Control a Realistic Goal?" Department of Agronomy, Iowa State University Extension Agronomy, 1996, 3 p.

[16] S. A. Gower, M. M. Loux, J. Cardina and S. K. Harrison, "Effect of Planting Date, Residual Herbicide, and PostEmergence Application Timing on Weed Control and Grain Yield in Glyphosate-Tolerant Corn (Zea mays)," Weed Technology, Vol. 16, No. 3, 2002, pp. 488-494. doi:10.1614/0890-037X(2002)016[0488:EOPDRH]2.0.C $\underline{\mathrm{O} ; 2}$

[17] M. M. Loux, A. F. Dobbels, W. G. Johnson, G. R.W Nice, T. T Bauman and J. M. Stachler, "Weed Control Guide 
for Ohio and Indiana," Ohio State University Extension Bulletin 789/Purdue Extension Pub No. WS16, 2008, 201 p.

[18] S. A. Payne and L. R. Oliver, "Weed Control Programs in Drilled Glyphosate-Resistant Soybean," Weed Technology, Vol. 14, No. 2, 2000, pp. 413-422. doi:10.1614/0890-037X(2000)014[0413:WCPIDG]2.0.C $\underline{\mathrm{O} ; 2}$

[19] J. L. Corbett, S. D. Askew, W. E. Thomas and J. W. Wilcut, "Weed Efficacy Evaluations for Bromoxynil, Glufosinate, Glyphosate, Pyrithiobac, and Sulfosate," Weed Technology, Vol. 18, No. 2, 2004, pp. 443-453. doi:10.1614/WT-03-139R

[20] L. C. Gonzini, S. E. Hart and L. M. Wax, "Herbicide Combinations for Weed Management in Glyphosate-Resistant Soybean (Glycine max)," Weed Technology, Vol. 13, No. 2, 1999, pp. 354-360.

[21] R. E. Nurse, A. S. Hamill, C. J. Swanton, F. J. Tardif, W. Deen and P. H. Sikkema, "Is the Application of a Residual Herbicide Required Prior to Glyphosate Application in No-Till Glyphosate-Tolerant Soybean (Glycine max)?" Crop Protection, Vol. 26, No. 4, 2007, pp. 484-489. doi:10.1016/j.cropro.2006.04.018

[22] L. E. Steckel, C. L. Sprague and A. G. Hager, "Common Waterhemp (Amaranthus rudis) Control in Corn (Zea mays) with Single Preemergence and Sequential Applications of Residual Herbicides," Weed Technology, Vol. 16, No. 4, 2002, pp. 755-761. doi:10.1614/0890-037X(2002)016[0755:CWARCI]2.0.C $\underline{\mathrm{O} ; 2}$

[23] J. Kovach, C. Petzoldt, J. Degni and J. Tette, "A Method to Measure the Environmental Impact of Pesticides," New York's Food and Life Sciences Bulletins, Vol. 139, 1992, pp. 139-146.

[24] J. Kovach, C. Petzoldt, J. Degni and J. Tette, "A Method to Measure the Environmental Impact of Pesticides," 2012. http:/www.nysipm.cornell.edu/publications/eiq/

[25] J. Fernandez-Cornejo, "Environmental and Economic Consequences of Technology Adoption: IPM in Viticul- ture," Agricultural Economics, Vol. 18, No. 2, 1998, pp. 145-155. doi:10.1016/S0169-5150(97)00054-6

[26] G. Edwards-Jones and O. Howells, "The Origin and Hazard of Inputs to Crop Protection in Organic Farming Systems: Are They Sustainable?" Agricultural Systems, Vol. 67, 2001, pp. 31-47. doi:10.1016/S0308-521X(00)00045-7

[27] C. R. Ziegler, D. W. Donahue, F. A. Drummond and S. N. Smith, "The Ecological Economics of Insecticide Use Associated with the Maine Potato Industry Based on a Producer Survey," American Journal of Alternative Agriculture, Vol. 17, No. 4, 2002, pp. 159-166. doi:10.1079/AJAA2002159

[28] P. R. Bradley, W. G. Johnson, S. E. Hart, M. L. Buesinger and R. E. Massey, "Economics of Weed Management in Glufosinate-Resistant Corn (Zea mays L.)," Weed Technology, Vol. 14, No. 3, 2000, pp. 495-501. doi:10.1614/0890-037X(2000)014[0495:EOWMIG]2.0.C $\mathrm{O} ; 2$

[29] W. G. Johnson, P. R. Bradley, S. E. Hart, M. L. Buesinger and R. E. Massey, "Efficacy and Economics of Weed Management in Glyphosate-Resistant Corn (Zea mays)," Weed Technology, Vol. 14, No. 1, 2000, pp. 5765.

doi:10.1614/0890-037X(2000)014[0057:EAEOWM]2.0. $\mathrm{CO} ; 2$

[30] L. P. Gianessi, "Economic and Herbicide Use Impacts of Glyphosate-Resistant Crops," Pest Management Science, Vol. 61, No. 3, 2005, pp. 241-245. doi:10.1002/ps.1013

[31] J. A. Ferrell and W. W. Witt, "Comparison of Glyphosate with Other Herbicides for Weed Control in Corn (Zea mays): Efficacy and Economics," Weed Technology, Vol. 16, No. 4, 2002, pp. 701-706. doi:10.1614/0890-037X(2002)016[0701:COGWOH]2.0. $\mathrm{CO} ; 2$

[32] Ontario Ministry of Agriculture, Food and Rural Affairs (OMAFRA), "Guide to Weed Control, Publication 75," Toronto, 2008, 379 p. 